\title{
Electrical transport in granular metals
}

\author{
Chongpu Zhai $^{1}$, Dorian Hanaor ${ }^{2}$, Gwénaëlle Proust ${ }^{1}$ and Yixiang Gan ${ }^{1, *}$ \\ ${ }^{1}$ School of Civil Engineering, The University of Sydney, Sydney, Australia \\ ${ }^{2}$ Institute for Material Science and Technologies, Technische Universität Berlin, Germany
}

\begin{abstract}
In this paper, we studied the frequency-dependent AC conductance of randomly packed stainless steel spheres by means of impedance spectroscopy. Two types of power-law behaviour have been observed: (a) at low frequencies, the dependence of the measured impedance on the applied load; (b) at high frequencies, the dependence of the impedance modulus on frequency. Under different loading conditions, the imaginary parts of the measured conductances exhibit respective peaks at critical frequencies, corresponding to the onset of conductance dispersion. Using these critical points as scaling parameters to normalize the measured conductance, results in the spectra from different loading levels collapsing onto a single master curve. Both the electron tunnelling and capacitive paths among particles contribute to the conduction in granular metallic media, resulting in well-characterized universal behaviour.
\end{abstract}

\section{Introduction}

The electrical conduction of granular materials is important in many engineering applications, such as powder metallurgy [1], thermoelectric systems [2], tactile sensing devices [3], and soil moisture content measurements [4]. Generally, these systems comprise assemblies of large numbers of conductive and/or insulating particles in contact. In order to evaluate the overall electrical properties of such systems, they can be considered as complex networks of randomly positioned electrical components, which can be regarded as resistorcapacitor (RC) networks [5-7]. Electrical responses of such RC networks subjected to alternating current (AC) have been investigated using experimental [8-10], numerical [11,12], and theoretical approaches [7,13,14]. These studies have shown that electrical characteristics, including conductivity, impedance, and permittivity exhibit power-law type dependences on the $\mathrm{AC}$ frequency, $\omega$, known as the 'universal dielectric response' (UDR) [15], within a certain range of frequencies. For example, the conductivity can be expressed as:

$$
\sigma^{\prime}(\omega)=\sigma_{d c}+A \omega^{n},
$$

where $\sigma_{d c}=\lim _{\omega \rightarrow 0} \sigma^{\prime}(\omega)$ is the direct current (DC) conductivity of the material, $A$ is a constant, and $n$ is the fractional exponent which is typically $0 \leq n \leq 1$. Both $A$ and $n$ are temperature dependent. Jonscher's UDR successfully describes most disordered dielectric systems when AC conductivity and permittivity show a dispersive region beyond a critical frequency. Moreover, it has been shown in a range of systems, from semiconductors to polymers, that the conductivity spectra collapse into a single master curve, suggesting the validity of the time-temperature superposition principle [15-17], which can be expressed by the following scaling law

$$
\sigma^{\prime}(\omega) / \sigma_{d c}=F\left(\omega / \omega^{*}\right),
$$

where $F$ is a temperature-independent scaling function and $\omega^{*}$ is the characteristic frequency corresponding to the onset of the conductivity dispersion, which in turn is a scaling parameter [15-17]. Temperature affects the density of charge carriers in ionic conductors or the electron hopping probabilities in granular metals [18]. This change of conduction properties between grains can actually be achieved also by varying the mechanical loading, which influences the inter-particle distance and contact area between particles, thus affecting the conduction behaviour [19-21]. These systems have been simulated using random $\mathrm{RC}$ networks and the electrical responses have been found to follow the universal power law [22-25]. This supports that the emergent scaling phenomenon develops from the network nature of the system $[15,22]$ without referring to element properties.

The aim of this paper is to examine the frequencydependent collective electrical responses of packed stainless steel grains, considered as conductor-insulator elements. We focus on the evolution of established networks under varying compressive loads. This study demonstrates the importance of understanding, at the element level, the origins of the intrinsic electrical characteristics of multi-phase materials or components which can be represented by a RC network.

\section{RC network formed by grain contacts}

In this paper, randomly packed conductive spheres are considered. The electrical characteristics of this system can be simplified to an electrical network formed 
by a large number of $\mathrm{RC}$ components in random positions. A single electrical contact between two grains can be represented by a resistor and/or a capacitor, depending on the inter-particle force and distance. The electrical conduction through isolated spheres is capacitive as the DC current could not tunnel through the interval. As for the slightly contacting spheres, a thin surface oxide layer acting as a resistive film with typical thickness ranging from 1 to $100 \mathrm{~nm}[26,27]$, tends to form and cover the metallic material. Consequently, these dielectric layers cause contacts to be nonconductive, under conditions of low loads, effectively acting as capacitors.

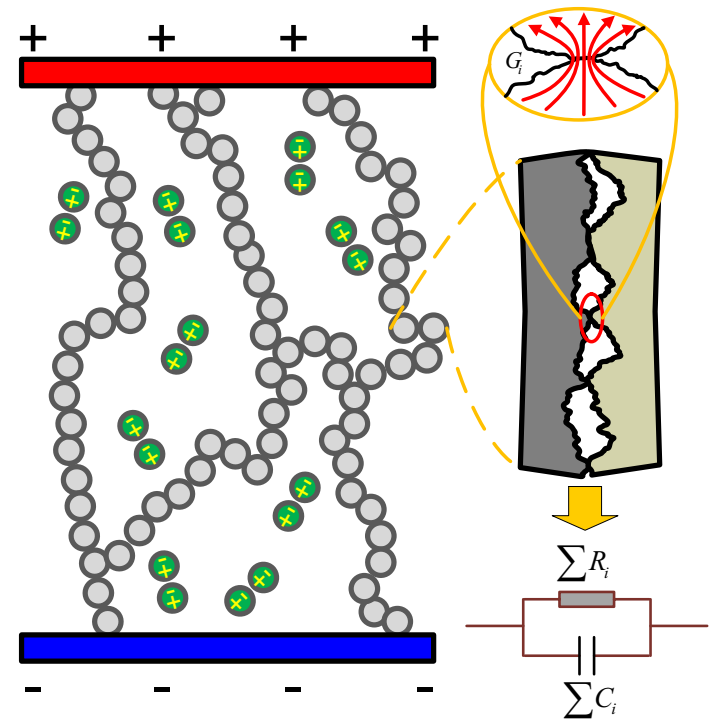

Fig. 1. Schematic of the electrical conduction through packed spheres. Typical percolating chains are indicated by connecting grey particles. Typical capacitors are shown by pairs of close but non-contacting spheres.

For contacting spheres, surface roughness plays an important role in the conduction properties [28]. As shown in Fig. 1, the contacting interfaces between grains tend to exhibit complex geometries and structures governing physical properties and interfacial phenomena [19] and giving rise to constriction resistance [29]. The constriction resistance due to the convergence and divergence of current flow at a single contact, results in a high resistance state. When two balls are compressed together with sufficient pressure, surface asperities deform and even penetrate the oxide layer thus forming small metal-to-metal contact patches. This leads to the low conduction of electrical current with electrons being transported in different ways depending on the size of contacting spots, i.e., Sharvin, Wexler, and Maxwell mechanisms $[21,25,29,30]$. When the radius of the contacting asperities, is smaller than the average electron mean free path, electrons can only travel ballistically across the micro-contacts [20]. Additionally, tunnelling through the oxide layer contributes to the conduction at this stage [21]. Under increasing compression, the numerous independent contacting spots increase in size, merging into larger contact areas [19]. When the effective radius of a contacting asperity becomes larger than the mean free path of electrons, Maxwell contact [31] will be the dominant transport mechanism, resulting in a relative low resistance. With further increasing interparticle force, large zones of metal-to-metal contact appear, likely establishing metal chains along several contacting grains, presenting inductive properties [25]. Noticeably, the inductive phase may not appear for metals with thick oxide layers even with high levels of compression [13]. On the other hand, the non-contacting zones at the interface act effectively as capacitors in parallel, which is also true for contacts without oxide layers. The contacting spheres can be then regarded as a resistor and a capacitor in parallel.

\section{Experimental setup}

In this paper, experiments were performed with commercial stainless steel ball bearings (Material: AISI 304; Precision Grade: G200) with diameters of $2 \mathrm{~mm}$. Spheres of $2400 \mathrm{~g}$ were randomly packed in a nonconductive cylinder (Ultra High Purity $\mathrm{Al}_{2} \mathrm{O}_{3}$, Taylor Ceramic Engineering, Australia) of $85 \mathrm{~mm}$ in height, $\mathrm{H}$, and with an inner diameter, D, of $98 \mathrm{~mm}$. The packed spheres were positioned between two metallic plates acting as electrodes. The impedance and phase angle were simultaneously measured using an impedance analyzer (Agilent, 4294A), with a measurement frequency ranging from $40 \mathrm{~Hz}$ to $20 \mathrm{MHz}$. The electrical responses were monitored under different loading levels, with each subsequent loading level being $4 / 3$ times that of the previous one, reaching a maximum load of $250 \mathrm{~N}$ (roughly 10 times of the self-weight of sphere assembly). Before each experimental sequence, the container was properly shaken to produce fresh contacts. The amplitude of the voltage applied to the systems was fixed to a low level with the peak-to-peak voltage value being $200 \mathrm{mV}$, in order to avoid voltage-induced surface modification [29]. For each given load, a one-minute equilibration time was given to minimize the variation of the measured impedance due to the creep deformation. It should be noted that the effects of the necessary cables and connections (which are in series with the sample under testing) have been subtracted through calibration processes.

\section{Results and discussion}

The typical measured impedance modulus of the studied granular system under different levels of compression is presented as a function of frequency in Fig. 2, with the phase angle shown in the insert. A larger load results in higher conductance and larger phase angle across the entire studied frequency range. The negative values of phase angle obtained indicate the capacitive properties. For relatively higher frequencies, a linear dependence in a log-log plot emerges over two orders of magnitude, which corresponds to power law dependence. For a certain range of frequencies, the absolute value of the exponent of the observed power-law function seemingly decreases with increasing pressure. We will come back to this point in the following discussion. 


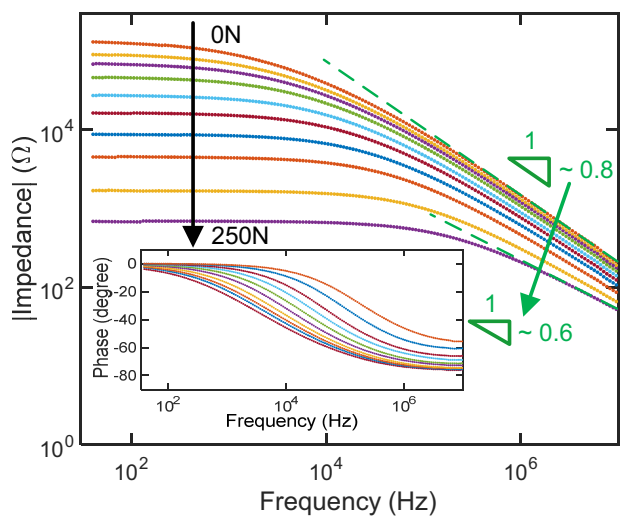

Fig. 2. Typical dependence of the measured impedance with respect to the frequency for the studied spherical grains. The phase angle variation with frequency is shown in the insert. Each individual curve corresponds to a given compression level with the arrow showing the increasing trend.

The relationships shown in Fig. 2 are attributed to the intricate electrical network formed by the elements, regardless of the physical or chemical properties of the material involved $[5,7,8]$. The frequency dependence of the conductance can be divided into three regions. At low frequencies, the network conductance is dominated by random percolation paths of resistors across the network. At intermediate frequencies, where the conductance of the capacitors and resistors become comparable, both types of components contribute to the network conductivity. In this frequency range, conductance rises as a power function of frequency. Importantly, the power law exponent is equal to the capacitive component proportion [11,22,32]. At high frequencies, the conductance of the capacitors would far exceed those of the resistors and thus these components can be regarded as short circuits. Consequently, the network conductance is determined by the random distribution of resistors, effectively bound together by shorting capacitors, reaching saturated values. However, the frequencies at this stage cannot be reached within the present methodology.

It should be noted that various mechanisms of surface structure evolution have been observed during electrical conduction through rough interfaces, including dielectric breakdown of oxide layers, localised current-induced welding, chemical disorder arising with random composition and oxidation processes in corrosive environments, and surface diffusion [26, 29]. These phenomena are, however, outside the scope of the present work.

The dependence of resistance on applied pressure, measured at low frequencies is presented in Fig. 3. Here the resistance, the real part of measured impedance, was averaged over numerous tests with error bars indicating the measurement range obtained at the selected measurement frequency $(40 \mathrm{~Hz})$. The non-dimensional electrical resistance of the sphere assembly decreases by several orders of magnitude under increasing stress. A larger applied load results in larger inter-particle force, thus a better conductance through the contact, therefore, the assembly. The dimensionless resistance and pressure can be used to estimate the contact properties between two grains.

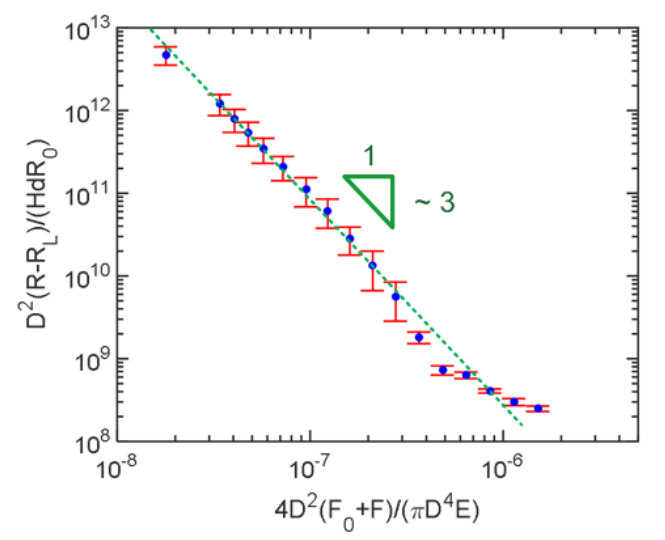

Fig. 3. Non-dimensional stress-dependent electrical resistance of two packed bed types under various loading conditions at a frequency of $40 \mathrm{~Hz}$. Here we assume the spheres are packed in layers. The contact resistance between spheres in each layer are considered to be in parallel and layers in series. $E$ is the Young's Modulus of the tested material (203 GPa), $D$ is the inner diameter of the container, $d$ the sphere diameter, $R_{L}$ is the total resistance of the cables and connectors, $R$ the measured resistance, $R_{0}$ the theoretical resistance of the bulk material for an identical volume as that of the packed bed, $F_{0}$ is the sum of half self-weight and the weight of the top cap and $F$ is the applied compressive force.

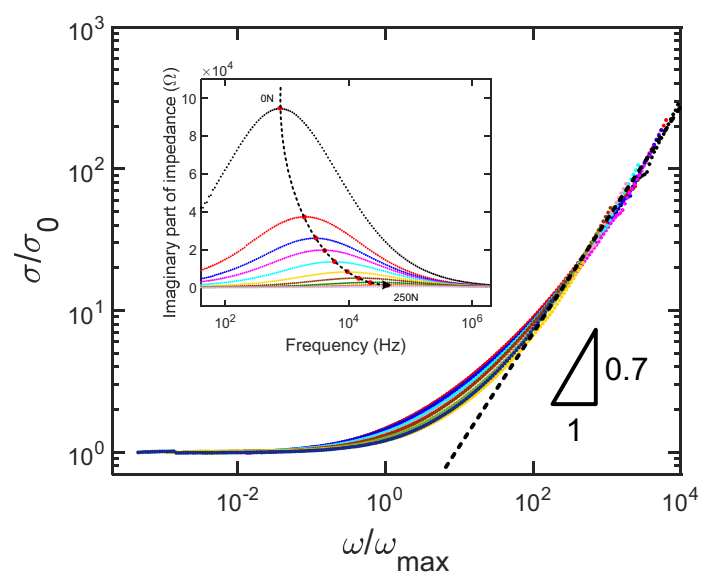

Fig. 4. Scaling plot of $\sigma / \sigma_{0}$ versus $\omega / \omega_{\max }$, with $\sigma$ being the module of conductance, $\sigma_{0}$ the conductance measured at a frequency of $40 \mathrm{~Hz}, \omega_{\max }$ the critical frequency corresponding to the onset of the conductivity dispersion. The insert shows the imaginary part of the measured impedance as a function of frequency with the red dots marking the peaks at critical frequencies. Each individual curve corresponds to a given compression with the arrow showing the increasing trend.

The electrical impedance across the considered frequency range has been analysed by plotting the imaginary part of impedance $\left(Z_{i}\right)$ versus frequency at different loading levels, as is shown in the insert of Fig. 4. This provides information on the dielectric processes 
taking place in the studied media. The critical frequency at the peak shifts to higher frequency with increasing load, indicating the existence of load-dependent relaxation process in the system. The critical frequencies along with the corresponding values of $Z_{i}$ are further included, as scaling parameters, in the conductance spectroscopy. The collapse of all the curves at different loading levels onto a single master curve has been observed, exhibiting an evident universal behaviour. Moreover, the exponent extracted from the dispersive region of the master curve has a value of around 0.7 . The explanation for this specific value needs to be traced back to the sphere contacts in the studied systems. According to Equation (1), the exponent can be directly related to the fraction of capacitive phases $[15,22,32,33]$. For our loading range, each pair of contacting spheres can be considered as a resistor and a capacitor in parallel, configuring the basic structure of a RC network, with the capacitor percentage being around $50 \%$. Additionally, the non-contacting and slightly touching sphere pairs, acting effectively as capacitors, add extra capacitive phases $(\sim 20 \%)$ into the RC network.

\section{Conclusion}

The scaling behaviour of AC electrical responses of granular stainless steel has been studied under increasing load. Both the resistive and capacitive paths among particles contribute to the conduction in granular metals, resulting in well-characterized universal behaviour. The real part of the measured impedance at low frequency follows a power law relationship with respect to the applied load. For various loading conditions, the frequency-dependent conductance is found to obey the emergent scaling power law, with a universal exponent value of about 0.7 , which tends to be unaffected by the applied compressive load.

\section{References}

1. R. M. German, Solar-Terrestrial Physics (Solnechno-zemnaya fizika), 568 (1996).

2. L. E. Bell, Science 321, 1457 (2008).

3. S. Stassi, V. Cauda, G. Canavese, and C. F. Pirri, Sensors 14, 5296 (2014).

4. S. R. Evett, J. A. Tolk, and T. A. Howell, Vadose Zone Journal 4, 1020 (2005).

5. K. Murphy, G. Hunt, and D. P. Almond, Philosophical Magazine 86, 3325 (2006).

6. P. Watts, W. Hsu, V. Kotzeva, and G. Chen, Chemical physics letters 366, 42 (2002).

7. J. Clerc, G. Giraud, J. Laugier, and J. Luck, Advances in Physics 39, 191 (1990).

8. M. Creyssels, E. Falcon, and B. Castaing, Physical Review B 77, 075135 (2008).

9. B. Castaing and C. Laroche, Europhysics Letters 65, 186 (2004).

10. S. Dorbolo, M. Ausloos, and N. Vandewalle, Physical Review E 67, 040302 (2003).
11. R. Bouamrane and D. P. Almond, Journal of Physics: Condensed Matter 15, 4089 (2003).

12. R. K. H. Galvão, S. Hadjiloucas, K. H. Kienitz, H. M. Paiva, and R. J. M. Afonso, Circuits and Systems I: Regular Papers, IEEE Transactions on 60, 624 (2013).

13. C. Grimaldi, P. Ryser, and S. Strässler, Journal of applied physics 92, 1981 (2002).

14. R. Kawakami Harrop Galvao, K. H. Kienitz, S. Hadjiloucas, G. C. Walker, J. W. Bowen, S. Figueredo Carreiro Soares, and U. Araujo, Dielectrics and Electrical Insulation, IEEE Transactions on 20, 995 (2013).

15. A. K. Jonscher, nature 267, 673 (1977).

16. B. Roling, Solid State Ionics 105, 185 (1998).

17. J. C. Dyre, P. Maass, B. Roling, and D. L. Sidebottom, Reports on Progress in Physics 72, 046501 (2009).

18. H. Bakkali, M. Dominguez, X. Batlle, and A. Labarta, Scientific Reports 6 (2016).

19. C. Zhai, Y. Gan, D. Hanaor, G. Proust, and D. Retraint, Experimental Mechanics 56, 359 (2016).

20. C. Zhai, D. Hanaor, G. Proust, L. Brassart, and Y. Gan, Extreme Mechanics Letters (2016).

21. L. Kogut and K. Komvopoulos, Journal of applied physics 95, 576 (2004).

22. D. Almond, C. Budd, M. Freitag, G. Hunt, N. McCullen, and N. Smith, Physica A: Statistical Mechanics and its Applications 392, 1004 (2013).

23. D. P. Almond, C. J. Budd, and N. J. McCullen, in Approximation Algorithms for Complex Systems (Springer, 2011), pp. 3.

24. D. P. Almond and C. Bowen, Physical review letters 92, 157601 (2004).

25. C. Zhai, D. Hanaor, G. Proust, and Y. Gan, in 2016 IEEE 62th Holm Conference on Electrical Contacts (IEEE, 2016), pp. 9.

26. G. Proust, D. Retraint, M. Chemkhi, A. Roos, and C. Demangel, Microscopy and Microanalysis 21, 919 (2015).

27. E. Kikuti, R. Conrrado, N. Bocchi, S. R. Biaggio, and R. C. Rocha-Filho, Journal of the Brazilian Chemical Society 15, 472 (2004).

28. J. Greenwood, British Journal of Applied Physics 17, 1621 (1966).

29. C. Zhai, D. Hanaor, G. Proust, and Y. Gan, Journal of Engineering Mechanics, B4015001 (2015).

30. A. Mikrajuddin, F. G. Shi, H. Kim, and K. Okuyama, Materials Science in Semiconductor Processing 2, 321 (1999).

31. R. L. Jackson, E. R. Crandall, and M. Bozack, in 2012 IEEE 58th Holm Conference on Electrical Contacts (Holm), (IEEE, 2012), pp. 1.

32. D. P. Almond, C. Bowen, and D. Rees, Journal of Physics D: Applied Physics 39, 1295 (2006).

33. K. Falconer, Fractal geometry: mathematical foundations and applications (John Wiley \& Sons, 2013). 